\title{
Improving the fatigue performance of porous metallic biomaterials produced by Selective Laser Melting
}

\author{
Brecht Van Hooreweder ${ }^{1 *}$, Yanni Apers ${ }^{1}$, Karel Lietaert ${ }^{2,3}$, Jean-Pierre Kruth ${ }^{1}$ \\ ${ }^{1}$ Department of Mechanical Engineering, KU Leuven, Leuven, Belgium \\ ${ }^{2} 3 D$ Systems - LayerWise NV, Leuven, Belgium \\ ${ }^{3}$ Department of Materials Engineering, KU Leuven, Leuven, Belgium
}

\begin{abstract}
This paper provides new insights into the fatigue properties of porous metallic biomaterials produced by additive manufacturing. Cylindrical porous samples with diamond unit cells were produced from Ti6Al4V powder using Selective Laser Melting (SLM). After measuring all morphological and quasi-static properties, compression-compression fatigue tests were performed to determine fatigue strength and to identify important fatigue influencing factors. In a next step, post-SLM treatments were used to improve the fatigue life of these biomaterials by changing the microstructure and by reducing stress concentrators and surface roughness. In particular, the influence of stress relieving, hot isostatic pressing and chemical etching was studied. Analytical and numerical techniques were developed to calculate the maximum local tensile stress in the struts as function of the strut diameter and load. With this method, the variability in the relative density between all samples was taken into account. The local stress in the struts was then used to quantify the exact influence of the applied post-SLM treatments on the fatigue life. A significant improvement of the fatigue life was achieved. Also, the post-SLM treatments, procedures and calculation methods can be applied to different types of porous metallic structures and hence this paper provides useful tools for improving fatigue performance of metallic biomaterials.
\end{abstract}

Keywords: biomaterials, Selective Laser Melting, orthopaedic implants, fatigue life

\section{INTRODUCTION}

Lattice structures are a particular type of porous structures that consists of a network of connected unit cells. A unit cell is defined as the smallest repeatable volume of a lattice structure, and is often constructed from beams or struts which form a three-dimensional framework. Widely used unit cell types include diamond, cubic, truncated cuboctahedron, etc., although currently also more complex types of unit cells are investigated, such as sheet gyroid and plane gyroid [1-3]. When a biocompatible material such as Ti6Al4V or CoCr is used to produce these porous structures, they can be considered porous metallic biomaterials.

A porous biomaterial is characterized by its parent material and by the unit cell properties such as type, size, and relative density. The latter is a measure for the fraction of solid material in the porous structure. It is clear that the mechanical properties of the porous biomaterial can be altered by changing the parent material and the type and/or properties of the unit cell. As a consequence, lattice

* brecht.vanhooreweder@kuleuven.be structures are highly suitable for bone substituting biomaterials and for orthopaedic implants, as a biomaterial with mechanical properties similar to those of bone is preferred for these applications. A closer match between the mechanical properties of bone and the implant can minimize risks associated with stress shielding [2]. In addition, the porosity of such biomaterials can offer a large interconnected volume for bone ingrowth.

Apart from biomedical applications, these porous structures are also suitable for structural components with increased strength- and stiffness-to-weight ratios. Applications in aerospace can benefit most from these kind of structures. Finite element software could for instance be used to identify locations in a structural part where the maximum stresses are low and where porous structures could be used to reduce the overall weight of the part. This is called structural or topological optimization and is a research field in itself [4]. 
The availability of Additive Manufacturing (AM) techniques such as Selective Laser Melting (SLM) and Electron Beam Melting (EBM) has drastically increased the potential of porous metallic structures during the last 15 years [5-8]. With these AM techniques it has become feasible to produce multiple unique complex lattice structures in a single production run, enabling economical mass customization. The Ti6Al4V porous structures studied in this work, are produced by SLM. SLM is a layer-wise material addition technique which allows production of complex 3D parts by selectively melting successive layers of metal powder onto each other, using the thermal energy supplied by a focused and computer controller laser beam [9]. Except for enabling the production of porous structures with complex geometries, SLM also offers the advantage of high accuracy and low material waste.

During the last years, quite some research efforts were made to improve the manufacturability of these complex structures by SLM [5-6]. The quasi-static compression behavior of metallic porous biomaterials produced by SLM is quite well understood and documented for samples with different relative densities and unit cells [10-13]. Analytical techniques have been successfully developed to predict this quasi-static behavior [14]. The fatigue behavior, on the other hand, is not yet fully understood. The effect of relative density and unit cell type on the compression fatigue strength was accounted for by Yavari et al. [15]. They showed that the SN-data for samples with one unit cell type and different relative densities approaches one single trend line when the applied stress is normalized with the compressive offset stress of the porous structure. This shows that meta-materials are structures when their smallscale properties are considered, but they behave as materials when their homogenized macroscopic properties are studied [16]. Similar findings were reported by F. Li et al. for porous titanium samples produced by diffusion bonding [17]. Most of these recent scientific works on fatigue of porous metals are based on previous works related to mechanical behavior of metal foams [18-21].

Few studies are available in which finite element simulations are used to predict the fatigue life of porous structures based on SN-data from the parent material and calculations of the local stresses in the struts [22,23]. Although the approach presented in these works is promising, the actual prediction of the fatigue life proves to be very difficult because of the following reasons:

a) The lack of fatigue data of the parent material (solid Ti6Al4V produced by SLM with the same process conditions as for the porous structures).

b) The difference in mechanical properties of a standardized solid test sample and a thin strut.

c) The complexity of including the role of other fatigue influencing factors such as (variations in) strut surface roughness, irregularities, material porosity, residual stresses and stress concentrations.
It is well known that as-built SLM parts are often characterized by high surface roughness, residual tensile stress at the surface and remaining porosities and imperfections. This typically leads to inferior fatigue resistance of as-manufactured parts compared to traditionally produced parts from the same material $[24,25]$. In the current study, the objective was not to deal with these fatigue influencing factors in FE-modeling, but rather to develop and use post-SLM techniques to reduce the effect of these factors and hence to improve the fatigue life of porous metallic biomaterials produced by SLM. In particular, the influence of stress relieving (SR), hot isostatic pressing (HIP) and chemical etching (CE) is studied on biomaterials with diamond unit cells. It is shown that a combination of HIP and CE significantly improves the fatigue life by changing the microstructure and by reducing surface roughness, material porosity and stress concentration factors. To quantify this increase in fatigue strength, a novel local stress approach is developed. SNcurves are constructed based on local strut stresses which are determined using analytical and numerical techniques. By doing so, the mass loss that occurs due to CE and which leads to reduced strut diameters, is efficiently taken into account. The presented local SN-approach for fatigue of porous metallic Ti6Al4V can also be applied to other porous structures and hence this paper provides useful tools for improving fatigue performance of metallic biomaterials.

\section{MATERIALS AND METHODS}

\subsection{Sample preparation}

Selective Laser Melting was used to manufacture 125 open porous structures from Ti6Al4V ELI powder according to ASTM F3001. Cylindrical samples with height $\mathrm{H}=15 \mathrm{~mm}$ and diameter $\mathrm{D}=10 \mathrm{~mm}$ were produced with their longitudinal axis perpendicular to the building plate. This sample geometry is in line with samples used in related scientific works $[5,11,14-17]$. Figure 1 shows the CAD-STL file of such a cylindrical sample constructed from diamond unit cells with size $\mathrm{a}=1 \mathrm{~mm}$. This way, at least 10 unit cells are present in all directions, which is important to avoid the size-effect in porous structures [26,27]. The average strut length $\mathrm{L}$ equals $0.433 \mathrm{~mm}$ and the angle between struts equals $109.5^{\circ}$. The strut diameter d depends on the applied surface treatment.

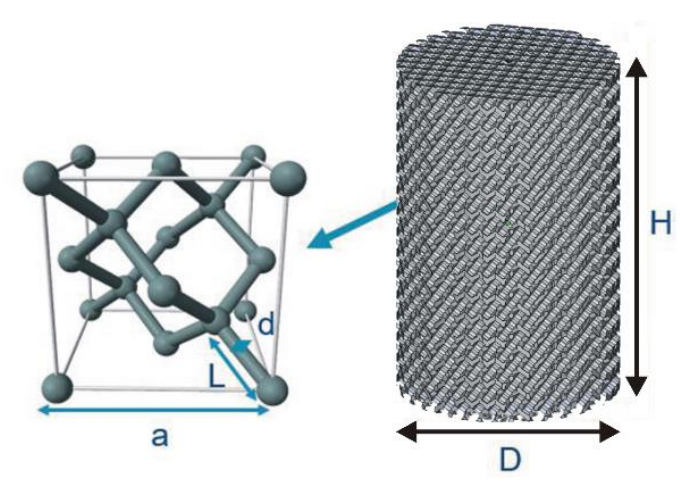

Figure 1. Dimensions of the diamond unit cell (left) and test samples (right) 
Diamond unit cells are used in this work since they are widely used and strongly isotropic. Biomedical implants typically have to withstand loading from multiple directions and therefore it is suggested to use isotropic unit cell designs [5]. The diamond unit cell design is also well suited for production by SLM. The inclination angle of all the struts with respect to the building platform equals $54.75^{\circ}$. This way, no support structure is required and the staircase effect is largely avoided, as shown by E. Yasa et al. in [28].

All samples were manufactured by 3D Systems on a ProX DMP 320 machine using optimized process parameters. A layer thickness of $30 \mu \mathrm{m}$ was used. The build chamber was flushed with Ar to create an inert atmosphere with an oxygen level below 25ppm. The samples were built on top of a solid titanium build plate from which they were subsequently removed using wire electrical discharge machining. After this, all samples were ultrasonically cleaned using demineralized water to remove unmolten powder particles.

The designed relative density of the specimens was $20 \%$. STL files were created using Magics software from Materialise (Leuven, Belgium). 3D Systems' DMP software was used for slicing and hatching of the STL file and for build preparation.

In this work, porous biomaterials in 4 different conditions were studied. First, samples in the as-built (AB) condition were analyzed to investigate the fatigue performance of samples with martensitic microstructure. Porous structures are often part of a larger implant and these almost always receive a heat treatment to reduce internal stresses before they are removed from the build plate. This means that the industrial relevance of the $\mathrm{AB}$ condition is low. From a scientific point of view, however, it is very interesting to include this condition in this study. Two different batches in $\mathrm{AB}$ condition were included in order to determine the influence of relative density on fatigue performance. Second, stress relieved (SR) samples were studied because of the industrial relevance of this condition. Residual stresses in porous structures produced by SLM are low, as the struts are free to deform. This means that the SR heat treatment does not change the stress state of a porous structure. However, porous structures often receive a SR heat treatment as part of a larger implant. In this treatment, the microstructure is changed and it is therefore important to include this condition in this study. Third, the Hot Isostatically Pressed (HIP) condition was included because of its importance for medical SLM parts and its effect on strut density and microstructure. Fourth, the inclusion of the Hot Isostatically Pressed and Chemically Etched (HIPCE) condition should allow a study of the influence of strut roughness on the mechanical performance. Chemical etching was applied on HIP samples since this is the most widely used heat treatment for biomedical implants. The reasoning above leads to the following batch names: $A B 1$, AB2, SR, HIP, and HIP-CE. For each batch, 5 samples were used for quasi-static testing and approximately 15-20 samples were used for fatigue testing. More information about SR, HIP and CE treatments for SLM Ti6Al4V samples can be found in [29] and [30].

\subsection{Morphological characterization}

The cross-sectional area and volume of every sample were accurately determined by measuring diameter $D$ and height $H$ with a caliper with $0.01 \mathrm{~mm}$ resolution. The mass $m$ of every sample was measured using a balance with $0.1 \mathrm{mg}$ resolution. Based on the theoretical density of Ti6Al4V $\rho_{T i}$ $=4.42 \mathrm{~g} / \mathrm{cm}^{3}$, the volume of solid material $V_{\text {solid }}$ could be calculated. The relative density $\rho_{\text {rel }}$ of all samples was then determined using Eq. 1.

$$
\rho_{\text {rel }}=\frac{V_{\text {solid }}}{V_{\text {tot }}}=\frac{m / \rho_{T i}}{H \pi D^{2} / 4}
$$

Previous studies have shown that these values of $\rho_{\text {rel }}$ determined based on dry-weighing closely correspond to relative densities determined using Archimedes measurements [5]. The porosity in the parent material (struts) was measured on five samples for every batch using Archimedes' principle in pure ethanol.

Scaffolds were embedded in cold mounting resin by vacuum infiltration for metallographic analysis (KEM90, ATM $\mathrm{GmbH}, \mathrm{Germany)}$. After grinding with $\mathrm{SiC}$ paper up to 4000 grit, the samples were polished with a silica suspension (OP$\mathrm{S})$. Etching with a mixture of $50 \mathrm{ml}$ demineralized water, $25 \mathrm{ml} \mathrm{HNO}_{3}$ and $5 \mathrm{ml} \mathrm{HF}$ revealed the microstructure of the samples. After imaging with a Nikon Eclipse MA100 microscope, the samples were polished again and re-etched with a $2 \%$ HF solution to study a possible alpha-case layer under the scaffold surface. An FEI XL30 Scanning Electron Microscope (SEM) was used to study the surface of the scaffolds at high magnification.

\subsection{Quasi-static testing}

An Instron 5985 machine with $30 \mathrm{kN}$ force cell was used for quasi-static compression testing according to ISO 13314. Before testing, the stiffness of the machine frame was measured and this was used for compliance correction. For sample testing, a compression load was applied with constant strain rate of $0.9 \mathrm{~mm} / \mathrm{min}$. The test was stopped at $70 \%$ compressive strain. For every batch, at least 3 samples were tested. From the compressive stress-strain curves the following properties were determined: the maximum stress $\sigma_{\max }$ and its corresponding strain $e_{\max }$, the plateau stress $\sigma_{p l}$ which is calculated as the arithmetic mean of the stresses between $20 \%$ and $40 \%$ strain, the quasi-elastic gradient $E_{Q E}$ which is the gradient of the straight line in the linear deformation region at the beginning of a compression test, and the compressive offset stress $\sigma_{\text {off }}$ which is the compressive stress at $0.2 \%$ plastic strain obtained from the quasi-elastic gradient. The offset stress is considered to represent the yield stress $\sigma_{y}$ of the porous structures to enable comparison with other studies.

It is important to remark that in line with all other studied on this topic, the stresses defined above are properties of the porous structure. Hence the yield, plateau and maximum 
stress values reported in this paper all refer to the virtual stresses acting on the porous structure, i.e. the applied load divided by the cross sectional area of the cylindrical sample. The term "local stress" is used to refer to the actual stress acting on the struts. The local stress is much more difficult to determine and therefore analytical and numerical techniques are used, as explained in the next sections.

\subsection{Fatigue testing}

An Instron Electropuls E10.000 with $10 \mathrm{kN}$ force cell was used to subject all fatigue samples to constant amplitude sinusoidal loading in compression-compression at fixed test frequency of $15 \mathrm{~Hz}$ and fixed load ratio $\mathrm{R}=0.1$. For every batch, at least 15 samples were tested. All experiments were force controlled and the stress based approach was used for fatigue analysis. SN-curves were constructed by testing at least two identical samples at one stress amplitude. Maximum stress values were determined at fixed percentages $(20 \%-90 \%)$ of the measured compressive offset stress. The failure criteria were defined as the moment where the test reached $4 \times 10^{6}$ cycles (run out), or when a sudden decrease in displacement occurred $(1 \mathrm{~mm} / \mathrm{s})$. During every test, the load, displacement and temperature were monitored. A compliance correction similar to the one used in quasi-static testing was applied to account for the deflection of the load frame. To allow lateral expansion of the samples during loading in compression, teflon paper of $0.3 \mathrm{~mm}$ thickness was placed between the sample and the hardened compression plates.

In line with all previous works, SN-curves were first constructed by plotting the global stress as function of the cycles to failure. In a next step, these global stress values were normalized with the compressive offset stress. In a final and novel step, the local normal tension stress in the struts was calculated and used to construct SN-curves in which the actual local fatigue behavior is represented.

\subsection{Analytical stress calculation}

For every porous structure that was tested in fatigue, Matlab was used to calculate the local normal stress occurring in the struts of the diamond unit cells. With this stress value, a local SN-curve could be constructed. These Matlab calculations are based on analytical approximations of the bending and axial compressive stresses which occur in the struts. Figure 2 shows an arbitrary strut of the diamond unit cell with hyperstatic boundary conditions similar to the free body-diagram presented in [14], and an approximation of the normal stress distributions which occur.

The strut force $F$ is function of the total compressive load on the sample $F_{\text {tot }}$ and of the number of nodes in the top cross section of the sample. As shown in Figure 1, a node at the top of the sample connects 2 struts and hence the strut force $F$ can be calculated as shown in Eq. 2.

$$
F=\frac{F_{t o t}}{\text { \#nodes } \cdot 2}
$$

The number of nodes in a circular cross section of a sample with diameter $D=10 \mathrm{~mm}$, unit cell size $a=1 \mathrm{~mm}$ and unit cell diagonal $a \sqrt{2} \mathrm{~mm}$ with 3 nodes can be approximated by 180 . This is shown below by calculating the number of nodes in a square sample with cross section $D x D$, and by multiplying this with the cross section ratio as shown in Eqs. 3-5.

$$
\begin{aligned}
& \text { \# } \text { nodes }_{\text {square }} \approx\left(\left(\frac{D}{a \sqrt{2}}-1\right) \cdot 2+1 \cdot 3\right)^{2} \\
& \frac{\text { rross section }_{\text {cylinder }}}{\text { cross section }_{\text {square }}}=\frac{\pi D^{2} / 4}{D^{2}}=\frac{\pi}{4} \\
& \text { \# } \text { nodes }_{\text {cylinder }} \approx\left(\frac{\pi}{4}\right) \cdot\left(\left(\frac{D}{a \sqrt{2}}-1\right) \cdot 2+1 \cdot 3\right)^{2} \\
& \approx 180 \text { (Eq. 5) }
\end{aligned}
$$

The axial compressive stress $S_{A}$ and the maximum bending stress $S_{B}$ can be calculated using strut force $F$, strut diameter $d$, strut length $L$, strut area $A$ and moment of inertia $I$ as shown in Eq. 6 and Eq. 7. The total stress $S$ in the strut is a superposition of $S_{A}$ and $S_{B}$ as indicated in Figure 2.

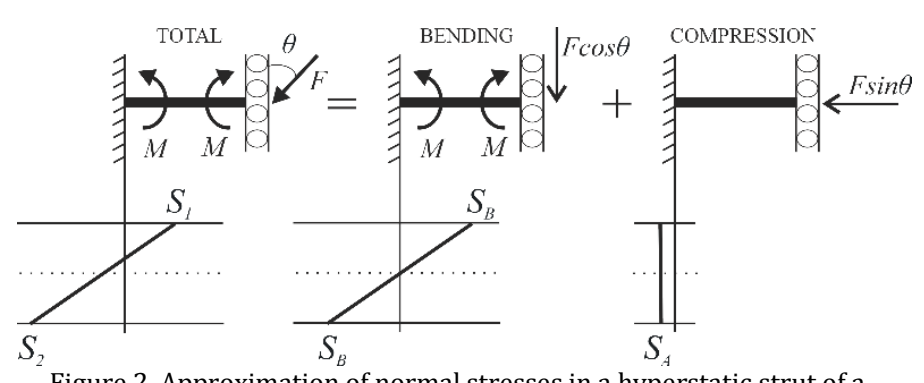

Figure 2. Approximation of normal stresses in a hyperstatic strut of a diamond unit cell

For mechanical fatigue, especially the maximum tensile stress component $S_{1}$ is of importance, even though it is smaller in amplitude than $S_{2}$. For these diamond unit cells with properties shown in Table 1 , the angle $\theta=35,26^{\circ}$ represents the orientation of the strut force $F$, as explained more in detail in [14].

$$
\begin{gathered}
S_{B}=\frac{M y}{I}=\frac{F L d \cos \theta}{4 I} \text { (Eq. 6) } \\
\text { with } I=\frac{\pi d^{4}}{64}, y=\frac{d}{2} \text { and } M=\frac{1}{2} F L \cos \theta \\
S_{A}=\frac{F \sin \theta}{A} \text { with } A=\frac{\pi d^{2}}{4} \text { (Eq. 7) } \\
S_{1}=S_{B}-S_{A}=F\left(\frac{16 L \cos \theta}{\pi d^{3}}-\frac{4 \sin \theta}{\pi d^{2}}\right)=F \cdot C_{\text {sample }}
\end{gathered}
$$

In this context, strut length $L$ and force angle $\theta$ are assumed to be constant and independent of the relative density and applied heat or surface treatment. The strut diameter $d$, on the other hand, is not constant and varies significantly with the relative density of the scaffold and hence also when a surface treatment is applied on the porous structure. 
This is taken into account by using Eq. 9, which is an analytical formulation developed by Gibson et al. to relate the relative density of the structure $\rho_{\text {rel }}$ to the strut radius $r$ and strut length $L$ [21]. In this formula, the effect of overlapping material in the vertex of connected struts is also taken into account. The geometrical constants $C_{1}$ and $C_{2}$ depend on the type of unit cell so this method can also be used for other unit cells such as truncated cuboctahedron and rhombic-dodecahedron. For the diamond unit cell used in this work $C_{1}$ equals 4.08 and $C_{2}$ equals 3.21 [21,22].

$$
\rho_{r e l}=C_{1}\left(\frac{r}{L}\right)^{2}-C_{2}\left(\frac{r}{L}\right)^{3}=1.02\left(\frac{d}{L}\right)^{2}-0.40\left(\frac{d}{L}\right)^{3}
$$

In this work, the average strut diameter $d$ is calculated for every sample by solving the third order Gibson formula for $L=0.433 \mathrm{~mm}$ and with the actual relative densities that were accurately measured for every sample as explained in Section 2.1. By doing so, the maximum local tensile stress $S_{1}$ $=S_{B}-S_{A}$ can be calculated for every tested sample by means of Eq. 8. This stress value can then be used to construct local stress-life diagrams, i.e. maximum local tensile stress in the struts as function of number of cycles to failure. Moreover, the constant $C_{\text {sample }}$ which takes the unit cell properties into account, can be used to normalize data from quasi-static compression tests, as explained in Section 3.1.

This analytical method for calculating the local stress in the struts of a diamond unit cell has the advantage of efficiency and speed. In addition, variations in relative density that are caused by process irregularities or by mass loss due to surface treatments can be accurately accounted for. For this reason, this analytical method is well suited to construct local stress-life curves for comparing fatigue strength before and after post-SLM treatments. It is, however, important to emphasize that the absolute stress values are only approximations of the true stress, since the following effects are not taken into account:

- Geometric non-linearities (i.e. $\theta=f(F)$ )

- Stress concentrations in the nodes

- Non-uniform cross-section of the struts

- $\quad$ Surface roughness and residual stress

- Internal material porosities

\subsection{Numerical stress calculation}

To gain more insights in the local stress distributions and to validate the analytical method, finite element calculations were performed on four samples with different relative densities. Struts were modelled as 1D quadratic Timonshenko beams with E-modulus of $114 \mathrm{GPa}$, poisson ratio of 0.342 and strut diameter as calculated by Eq. 9. The
FE-mesh was generated using 3-matic (Materialise NV) and further processing was performed in Abaqus/CAE 6.14. Boundary conditions were applied such that the bottom surface of the sample was restricted in all DOF, while the nodes at the top surface were connected and allowed to translate in the vertical loading direction. In a final step, the nodal forces, nodal displacements and the maximum normal tensile stresses were calculated and compared with results from the analytical method. This numerical approach uses the same assumptions as those used in the analytical approach mentioned in Section 2.5.

\section{RESULTS AND DISCUSSION}

\subsection{Sample properties}

Table 1 indicates the average relative densities and strut porosities for the different sample conditions as well as the properties resulting from the quasi-static compression tests.

The difference in relative densities between $A B 1, A B 2, S R$ and HIP samples is due to small variations in building of these samples. Not all batches were manufactured in the same production run and variations in relative density can occur due to minor differences in effective laser power, powder characteristics, etc. The much lower relative density of $31.12 \%$ for the HIP+CE batch is caused by the removal of material during chemical etching. As expected, the relative density greatly influences the mechanical properties. This can clearly be seen when comparing the results for the $\mathrm{AB} 1$ and $\mathrm{AB} 2$ batches, which only differ in relative density. Higher relative densities lead to higher strength and stiffness and so the difference in relative density makes it difficult to study the influence of post-build surface and/or heat treatments on the mechanical performance.

It should be noticed that variation of all properties mentioned in Table 1 between samples of the same batch $(\mathrm{AB}, \mathrm{SR}$, etc. ) is very low. The porosity in the struts is acceptable and decreases further when a HIP treatment is applied, as expected. The reason for the lower porosity of the SR condition is not clear since stress relief does not involve any pressure on the sample and hence porosities should not be influenced by this treatment. A possible reason for this value is the well-known inaccuracy of the Archimedes method to measure small $(<1 \%)$ levels of porosities [31].

Figure 3.A shows the compression-compression curves for three representative samples of the $\mathrm{AB}, \mathrm{SR}$ and HIP condition with different relative densities. As mentioned before, no clear conclusions can be drawn from this data.

\begin{tabular}{|c|c|c|c|c|c|c|}
\hline & $\rho_{\text {rel }}[\%]$ & Poros. [\%] & $E$ [GPa] & $\sigma_{\max }[\mathrm{MPa}]$ & $\sigma_{\mathrm{y}}[\mathrm{MPa}]$ & $\sigma_{\mathrm{pl}}[\mathrm{MPa}]$ \\
\hline AB1 & $34.42 \pm 0.50$ & $1.59 \pm 0.17$ & $4.301 \pm 0.131$ & $118.68 \pm 4.79$ & $90.11 \pm 2.59$ & $71.98 \pm 3.32$ \\
\hline AB2 & $37.42 \pm 1.32$ & $1.19 \pm 0.23$ & $4.921 \pm 0.425$ & $128.00 \pm 10.48$ & $101.50 \pm 7.32$ & $74.24 \pm 1.77$ \\
\hline SR & $34.31 \pm 0.74$ & $0.92 \pm 0.06$ & $4.941 \pm 0.172$ & $117.73 \pm 7.98$ & $103.59 \pm 5.19$ & $80.01 \pm 0.62$ \\
\hline HIP & $39.93 \pm 0.92$ & $0.60 \pm 0.05$ & $6.568 \pm 0.245$ & $146.85 \pm 1.69$ & $118.97 \pm 1.33$ & $133.78 \pm 2.10$ \\
\hline HIP+CE & $31.12 \pm 1.52$ & $0.73 \pm 0.06$ & $4.176 \pm 0.528$ & $88.64 \pm 10.44$ & $79.19 \pm 8.03$ & $97.70 \pm 14.33$ \\
\hline
\end{tabular}

Table 1. Average morphological and mechanical properties of all batches. 
However, the analytical local stress method described in Section 2.5 can be used to account for the differences in relative density.
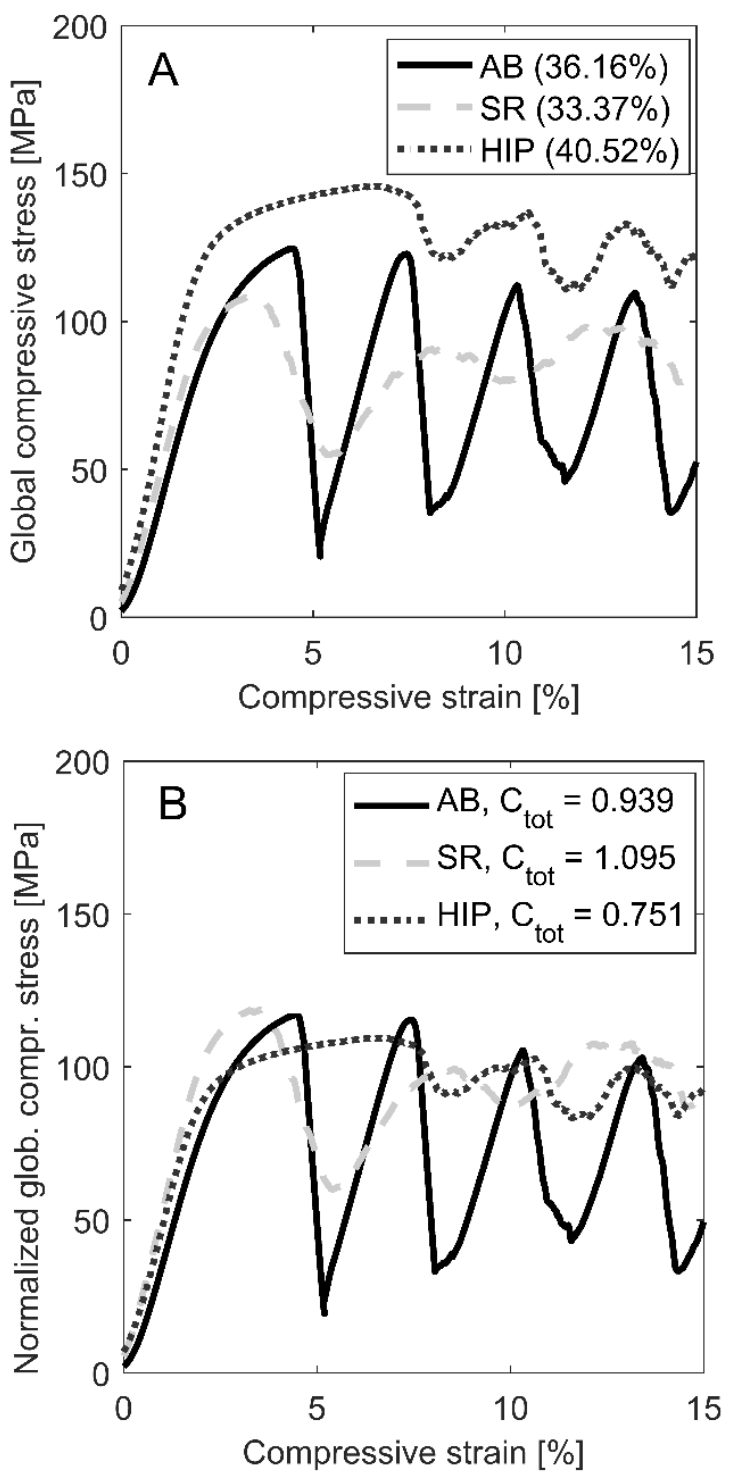

Figure 3. Quasi-static compression-compression behavior for AB, SR and HIP samples using global and normalized global stresses between 0 and $15 \%$ compressive strain

In Figure 3.B, all global stress values are normalized to global stress values that correspond to a sample with an arbitrary relative density of $35 \%$. This is done by calculating the average strut diameter $d$ of the representative AB, SR and HIP samples and of a reference sample with relative density of $35 \%$, by using the measured relative densities of these samples together with Eq. 9. In a next step, Eq. 8 is used to calculate $C_{\text {sample, }}$, which relates the strut force $F$ to the local normal tensile stress $S_{1}$. These results are summarized in Table 2. In a last step, these coefficients $C_{\text {sample }}$ are normalized to $C_{\text {tot }}$ and used to scale the global stress values. The HIP+CE condition does not significantly deviate from the HIP condition and is not plotted in Figure 3.B. for increased readability.

\begin{tabular}{|l|l|l|l|l|}
\hline & Ref & $\mathrm{AB}$ & $\mathrm{SR}$ & $\mathrm{HIP}$ \\
\hline$\rho_{\text {rel }}[\%]$ & 35 & 36.16 & 33.37 & 40.52 \\
\hline$d[\mathrm{~mm}]$ & 0.2968 & 0.3028 & 0.2883 & 0.3251 \\
\hline$C_{\text {sample }}\left[\mathrm{x} 10^{6}\right]$ & 60.530 & 56.844 & 66.304 & 45.453 \\
\hline$C_{\text {tot }}$ & 1 & 0.939 & 1.095 & 0.751 \\
\hline
\end{tabular}

Table 2. Constants for normalizing quasi-static data

By using this normalized data, the effect of the relative density can be correctly accounted for and hence the real influence of the applied post-build treatment on the quasistatic properties can be studied. From Figure 3.B, the following can be concluded:

a) When a sample is considered as a stack of horizontal unit cell layers which are loaded in compression, the AB sample fails in a brittle way by successive collapse of unit cell layers along $45^{\circ}$ crush bands. This is in line with quasi-static failure mechanisms reported in literature [32,33]. First the stress increases to a maximum at which one layer fails. This is followed by a stress drop which corresponds to the sudden brittle collapse of that layer, after which the next layer starts to deform, etc. This successive failure of layers for $\mathrm{AB}$ samples, and the high release of energy at each stage of the collapse, is clearly visible from the cyclic stress state in Figure 3. For the SR and HIP conditions, this cyclic stress phenomenon is much more damped, indicating that the sample deforms more plastically. This is in line with other studies which reported that in the AB-condition, bulk SLMTi6Al4V typically has high strength and low ductility compared to SR and HIP $[34,35]$.

b) The maximum stress for $\mathrm{AB}$ and $\mathrm{SR}$ samples is equal and higher than for HIP samples.

c) The strain at fracture of one layer is higher for HIP than for $\mathrm{AB}$ and SR samples.

d) The stiffness and yield strength of SR and HIP samples is higher than for $A B$ samples.

These last three findings are in line with a recent study by Wauthle et al., in which a smaller set of samples was produced in one production run without significant differences in relative densities between $A B, S R$ and HIP samples [29]. This indicates that the local stress method developed in this work, and applied for normalizing the quasi-static data in Figure 3, is a valid method to account for differences in relative densities when analyzing large (fatigue) sample sets with inherent variation in relative density. Furthermore, based on these results, the SR and HIP treatments are expected to increase the fatigue performance of porous structures as these samples deform less brittle than the $\mathrm{AB}$ samples. This can be inferred from the smaller stress drops for SR and HIP samples in Figure 3, which indicates a more ductile strut deformation [36]. 

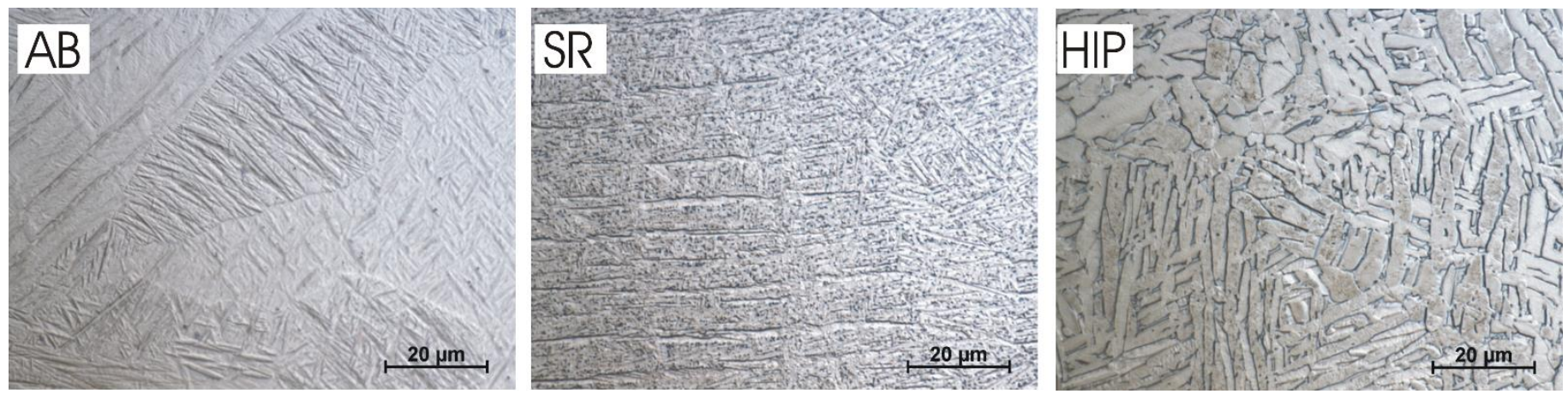

Figure 4. Microstructures of As-Built, Stress-Relieved and HIPed fatigue samples

The improved ductility of the SR and HIP samples over AB samples is caused by a change of the microstructure during these post-build heat treatments. Figure 4 shows the microstructures of a polished and etched strut of an as-built (AB), stress-relieved (SR) and hot isostatically pressed (HIP) fatigue sample. These microstructures are in-line with previous studies [29,34]. In the AB condition, an acicular martensitic $\alpha^{\prime}$-phase is present. During the SR treatment, the $\alpha$ martensitic phase transforms into $\alpha$ (white) and $\beta$ (black) by V expulsion [34]. The HIP treatment also creates the $\alpha$ and $\beta$ phases but the grains are much coarser than for the SR samples. This is caused by the higher temperature of the HIP treatment compared to SR. From these microstructures, it is clear that the transformation of the martensite and grain growth are responsible for the higher ductility of the SR and HIPed samples, compared to the more brittle $\alpha^{\prime}$-phase in the $\mathrm{AB}$ condition.
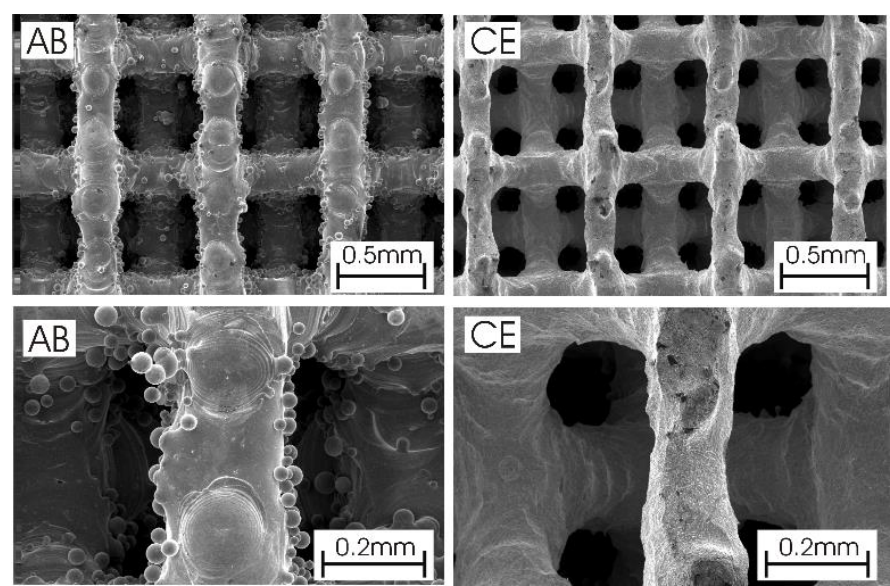

Figure 5. SEM image of the top surface of as-built (left) and chemically etched (right) fatigue samples

Apart from the microstructure of the parent material, the surface quality is expected to have a major influence on the cyclic mechanical performance as well. It is well known that additive manufactured materials are characterized by high surface roughness and that this is detrimental for the fatigue behavior.
In Figure 5, a high magnification SEM image of the top surface of a representative $\mathrm{AB}$ and HIP-CE fatigue sample is shown. For the $A B$ condition, the high surface roughness of the struts and the attached unmolten powder particles are clearly visible. The CE sample has smooth, slightly curved struts which are thinner in the middle and more pronounced at the nodes. As shown in Section 3.2, this smooth surface, in combination with an appropriate heat treatment for increased material ductility will improve the fatigue performance of these biomaterials. In addition, Figure 5 shows that the CE treatment also removes all the unmolten powder particles. This is beneficial for the use of these biomaterials as implants, as the risk of sintered particle release is removed. Also, the CE treatment results in overall thinner struts which explains the lower relative density shown in Table 1 and which motivates the use of a local stress method for the analysis of the mechanical fatigue behavior.

\subsection{Fatigue behavior}

In general, fatigue cracks originate from irregularities or imperfections at the surface or in the material which are subjected to cyclic loading of which the tensile stress component is most important. In this work, cyclic compression-compression loading is applied to open porous samples. The reason for the failure of these samples in fatigue is that the compressive loading and the global compressive stresses described in this work are properties of the cylindrical structure, and not of the struts. When looking at the fatigue samples as structures, it becomes clear that the struts or beams are actually loaded in a combination of cyclic bending and cyclic axial compressive stresses, as shown in Figure 2.

The cyclic loading of the structure in compressioncompression leads to both compressive and tensile stresses in the struts. It is most likely that these tensile stress components cause the successive fatigue failure of the struts, ultimately leading to fracture of the porous sample. 
Figure 6 shows three ways of presenting SN-data of open porous biomaterials, in this case for two as-built sample sets with different relative densities (AB1 and AB2).

1) In the first method (Figure 6A), the global compressive stress is used. This stress is calculated by dividing the applied force by the cross sectional area of the sample and hence the relative density or the strut diameter is not taken into account. These SN-curves therefore represent the behavior of the biomaterial, with increased fatigue behavior for increased relative density (i.e. larger strut diameter). The positive effect of increased relative density on fatigue life shown in Figure 6A corresponds with findings reported in other works on lattice structures $[5,13,14]$ and metallic foams $[18,19]$.

2) In the second method (Figure 6B), the applied compressive stress in normalized with the compressive offset stress of a similar sample with approximately identical relative density. These $\mathrm{SN}$-curves therefore represent the normalized fatigue behavior of the biomaterial. This method was also used by Wauthle et al. [5], Yavari et al. [15] and by Li et at. [17]. The microstructure, relative density, etc. directly influence the compressive offset stress of the sample and are hence indirectly taken into account. The SN-curves for AB1 and AB2 approach a single trend which corresponds more or less with literature data from similar SLM-Ti6Al4V samples [5]. The disadvantages of this method are: (i) no physical stress plotted on the Y-axis, (ii) representative additional sample needed for every sample condition and for every load orientation, (iii) limited accuracy, i.e. no perfect fit, and (iv) does not account for variability within one sample set.

3) In the third method (Figure 6C), the analytical approximation of the maximum local tensile stress $S_{1}$ in the struts of all the samples is used as explained in Section 2.5. In this case, the SN-curves represent an approximation of the actual fatigue behavior of the parent material, taking into account the strut diameter and the maximum stress state near the nodes of every single sample. This method shows improved accuracy over the first two methods. SNdata of $A B 1$ and $A B 2$ corresponds very well to a straight line on a $\log$-log scale. The coefficient of variation $R^{2}$ equals $99.8 \%$ and even data points from one sample set nicely coincide, e.g. the two top data points from AB2.

It is clear that the local stress method developed in this work can be used to compare the fatigue behavior of different sample sets (e.g. AB, SR, HIP, HIP+CE) while accurately taking into account the possible differences in relative density due to manufacturing variabilities and/or due to material removal by chemical etching. The absolute value of the maximum local stress is, however, still an approximation of the actual stress, since not all influencing factors are taken into account (see Section 2.5).
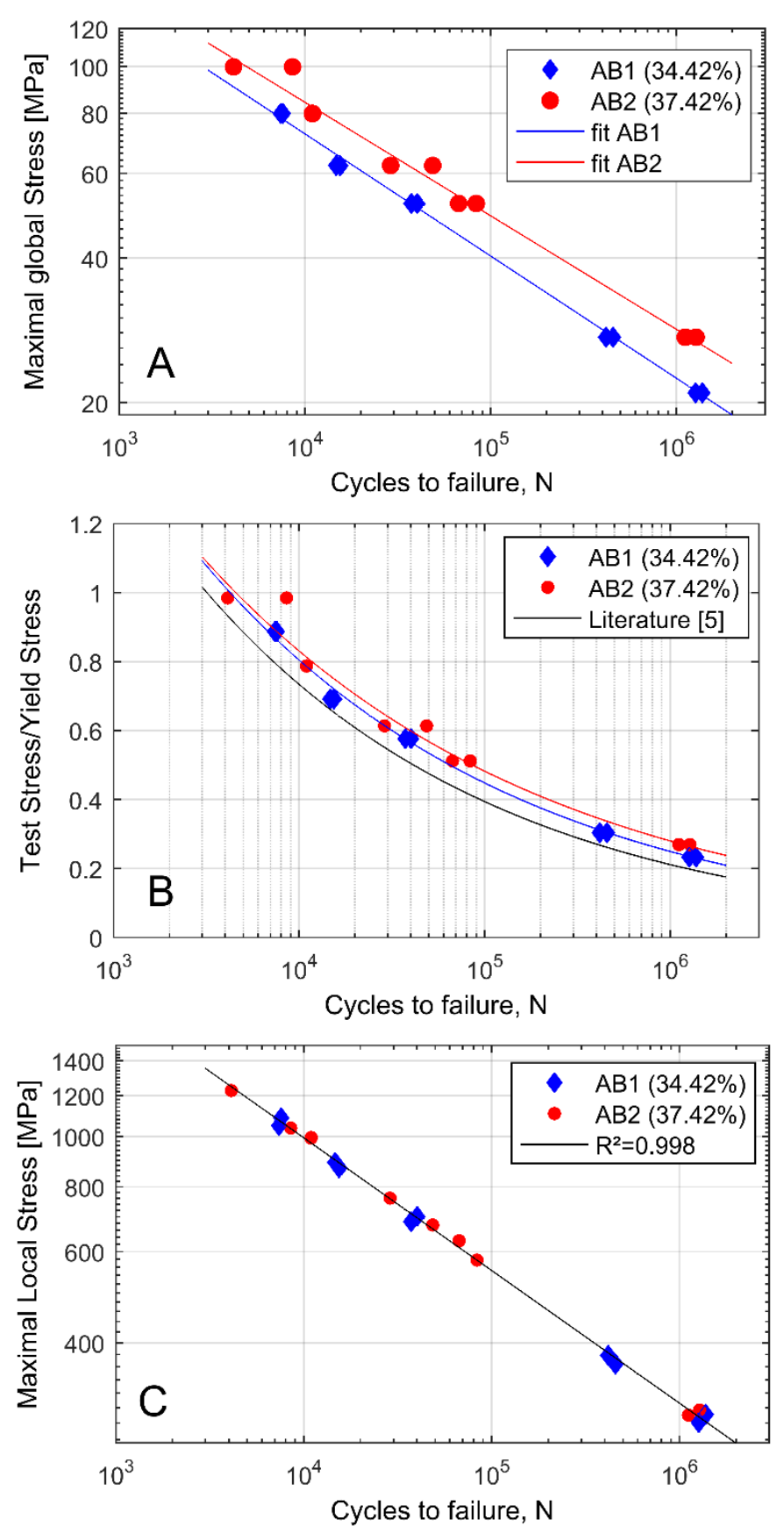

Figure 6. SN-curves of as-built sample sets with different relative densities: global stress method (A), normalized global stress method from [5] (B) and local stress method (C)

Figure 7 shows the combined fatigue data of all sample conditions on a log-log scale after applying the local stress method to account for the strut diameters of all samples. It is clear that the developed post-SLM heat and surface treatments have a positive effect on the fatigue life, especially in the high cycle region.

The SR and HIP treatment seem to result in an almost identical improvement of the fatigue life. This effect is mainly caused by the change in microstructure. The $\alpha^{\prime}$ martensitic phase of $A B$ samples transforms into an $\alpha+\beta$ phase for SR and HIP samples which leads to improved ductility and hence improved fatigue behavior. 


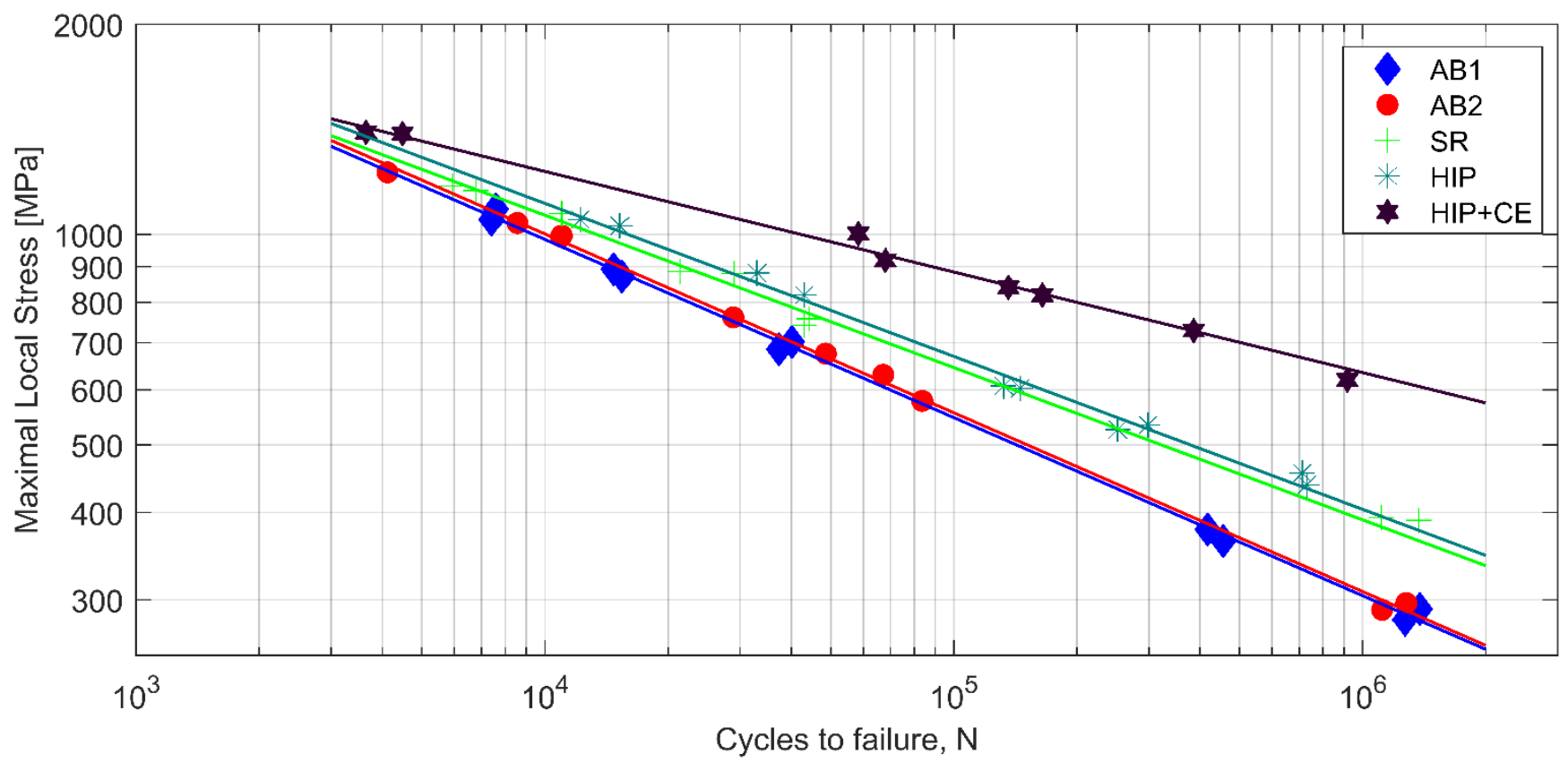

Figure 7. Influence of post-build treatments on fatigue behavior of SLM Ti6Al4V open porous biomaterials

The coarser microstructure of the HIP samples compared to the SR samples does not seem to have a significant influence. Also, the overall porosity in the struts is very low, and hence hot isostatic pressing only improves the fatigue performance by a very small amount compared to stress relieving. In addition, Figure 7 confirms the statement from Section 2.1 that residual stresses in porous structures are small and that consequently SR and HIP treatments do not change the stress state of a porous structure. If residual stresses would be present in $\mathrm{AB}$ samples and to a lesser extent in SR and HIP samples, the respective SN-curves would show a parallel offset. This is clearly not the case in Figure 7. The fatigue performance of HIP+CE samples is significantly better than AB, SR and HIP samples. This is due to the smooth surface, the reduced surface irregularities and the reduced stress concentration factor near the nodes of the unit cells. It is known that the influence of surface roughness is especially noticeable in the high cycle fatigue region which explains the slope of the HIP+CE curve [37].

\subsection{Numerical stress calculation}

Table 3 shows the calculated strut force $F$ and the maximum tensile stress in the strut $S_{1}$ as calculated by both the analytical and numerical methods for four samples with given strut radius $r$ and total load $F_{\text {tot. }}$.

\begin{tabular}{|l|l|l|l|l|l|l|}
\hline & $\begin{array}{l}r \\
{[\mu \mathrm{m}]}\end{array}$ & $\begin{array}{l}F_{\text {tot }} \\
{[\mathrm{N}]}\end{array}$ & $\begin{array}{l}F \\
{[\mathrm{~N}]} \\
\text { Analyt. }\end{array}$ & $\begin{array}{l}F \\
{[\mathrm{~N}]} \\
\text { FEM }\end{array}$ & $\begin{array}{l}S_{1} \\
{[\mathrm{MPa}]} \\
\text { Analyt. }\end{array}$ & $\begin{array}{l}S_{1} \\
{[\mathrm{MPa}]} \\
\text { FEM }\end{array}$ \\
\hline $\mathrm{a}$ & 148.4 & 2164.3 & 6.01 & 5.50 & 364 & 362 \\
\hline $\mathrm{b}$ & 145.9 & 4915.8 & 13.66 & 12.49 & 872 & 865 \\
\hline $\mathrm{c}$ & 147.6 & 6358.8 & 17.66 & 16.15 & 1088 & 1080 \\
\hline $\mathrm{d}$ & 152.0 & 7874.9 & 21.88 & 20.02 & 1228 & 1221 \\
\hline
\end{tabular}

Table 3. Strut force $F$ and strut stress $S_{1}$ calculated by analytical and numerical techniques
It can be seen that the differences in the results between the analytical and the numerical method are rather small and acceptable. This proves that, given the assumptions made in Section 2.5 and 2.6, the analytical procedure developed in this work can be successfully applied for calculating the strut force $F$ and the strut stress $S_{1}$ in porous metallic biomaterials with diamond unit cells and by accounting for different strut diameters. The analytical procedure is fast and straightforward, it provides insights in the loading of the struts and it is well suited for the purpose of analyzing the influence of post-built treatments on the fatigue behavior. The numerical procedure is more advantageous for complex shaped unit cells and for taking into account notch effects, non-linearities, etc.

\subsection{Fracture surface analysis}

In the diamond cell biomaterials studied in this work, the applied macro scale cyclic compressive loading results in a cyclic bending stress $S_{B}$ and a cyclic normal compressive stress $S_{A}$ applied to the constituent struts. The distribution of these stresses through the diameter of the struts is shown in Figure 2. As explained in Section 2.5, the resulting cyclic tensile stress component $S_{1}$ is most critical for mechanical fatigue. The mechanism of failure in the struts is hence crack initiation and crack propagation under tension-tension loading as a result of the fluctuating bending moment. The critical location for crack nucleation and initiation is hence located at the top of the struts and near the nodes where the tensile bending stress is maximum. This is confirmed by analyzing an $A B$ sample after eight hours of cyclic loading in the SEM. Figure 8 clearly shows fractured struts near the nodes of the top surface of the sample. This failure location corresponds with the predicted failure mechanisms and with the location of maximum tensile stress. 


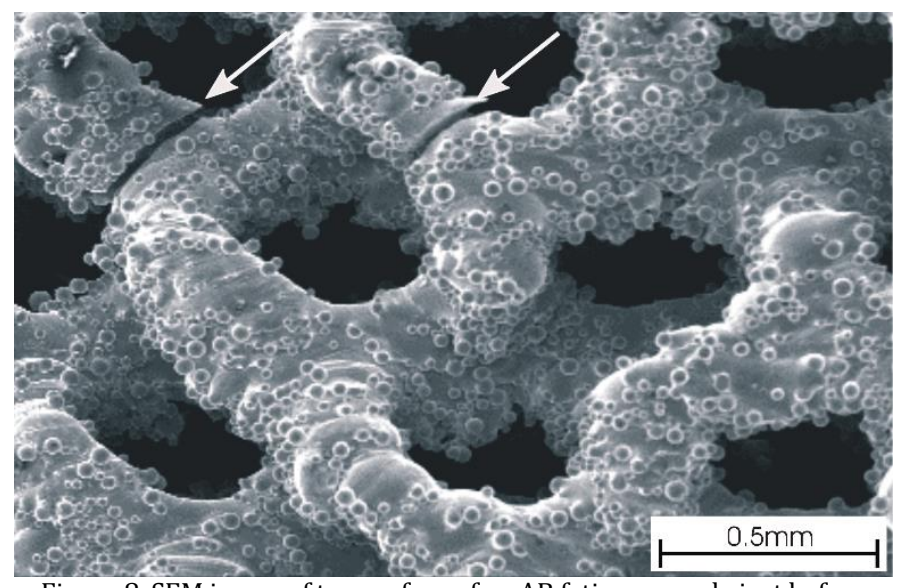

Figure 8. SEM image of top surface of an AB fatigue sample just before final failure

All fatigue samples failed by brittle fracture and no plastic deformation was measured during testing. Figure 9 shows a typical $A B$ fatigue sample before (left) and after (right) fatigue testing. The plane of fracture is orientated at approximately $45^{\circ}$ with respect to the loading direction. This corresponds to the $45^{\circ}$ crush bands that were found during quasi-static loading as described in Section 3.1. For quasi-static loading, horizontal "layers" of unit cells where successively crushed along $45^{\circ}$ bands while for cyclic loading, the sample fails in a brittle way along $45^{\circ}$. No differences were found in failure mechanisms between $A B$, $\mathrm{SR}, \mathrm{HIP}$, or HIP+CE samples. In addition, the failure mechanism corresponds with the findings reported in [13] for similar porous biomaterials.
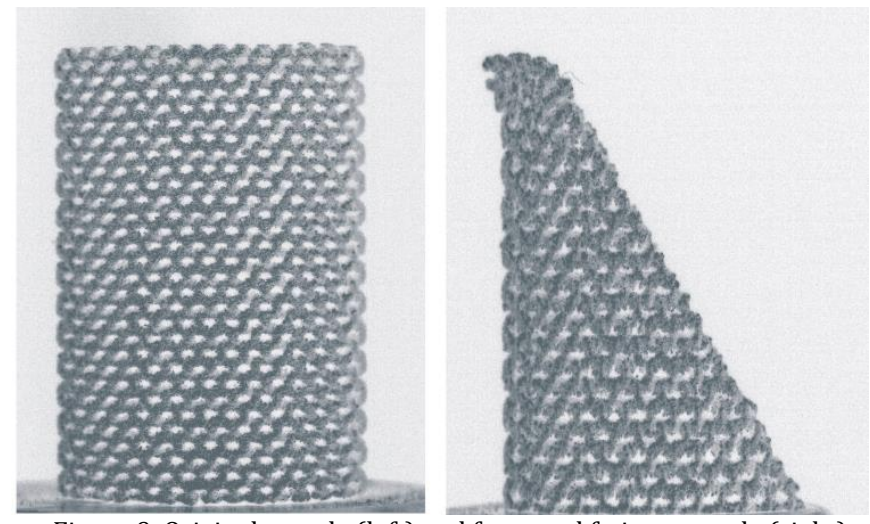

Figure 9. Original sample (left) and fractured fatigue sample (right) with $D=10 \mathrm{~mm}$ and $H=15 \mathrm{~mm}$

\section{CONCLUSIONS}

The availability of Additive Manufacturing techniques such as Selective Laser Melting (SLM) has significantly increased the potential and the use of porous metallic biomaterials for implants during the last years. However, the fatigue behavior and failure mechanisms of these structures are not yet fully understood. In this study, the influence of three post-built treatments, namely stress relieving (SR), hot isostatic pressing (HIP) and chemical etching (CE), on the fatigue life of Ti6Al4V diamond like structures was investigated. An efficient analytical method was developed to account for the differences in relative densities of those samples and to study the fatigue behavior based on the local state of stress occurring in the struts of the diamond unit cells. This analytical method was successfully validated experimentally and numerically, and performs well compared to the normalized global stress approach currently used in literature. In a next step, this local stress method was used to compare the fatigue performance before and after the developed post-SLM treatments. The results show that the SR and HIP treatments improve the fatigue life to a certain extent, while HIP+CE significantly improves the fatigue properties, especially in the high cycle region. The reason for this is the more ductile microstructure and the reduced stress concentration factor near the nodes of the unit cells, which are the critical points for crack initiation. The developed post-SLM treatments, procedures and calculation methods can be applied to different types of porous biomaterials with different unit cell designs and hence this paper provides useful tools for improving fatigue performance of porous metallic biomaterials. In addition, also the influence of other biofunctionalizing surface treatments on the mechanical behavior of porous biomaterials can be studied efficiently using the methods described in this work.

\section{Acknowledgements}

This research was supported by FWO (Fonds wetenschappelijk Onderzoek) Vlaanderen [grant number FW0-12S4615N] and by the agency for Innovation by Science and Technology (IWT) of the Flemish government through a Baekeland mandate [grant number IWT140257].

\section{References}

[1] K. Mediaswanti, C. Wen, E.P. Ivanova, C.C. Berndt, F. Malherbe, V. Pham, J. Wang, A review on bioactive porous metallic biomaterials, J. Biomim. Biomater. Tissue Eng. 18 (2013) 2.

[2] B.D. Ratner, A.S. Hoffman, F.J. Schoen, J.E. Lemons, Biomaterials science: an introduction to materials in medicine, third ed., Academic press, UK, 2013.

[3] C. Yan, L. Hao, A. Hussein, D. Raymont, Evaluations of cellular lattice structures manufactured using selective laser melting, Int. J. Mach. Tool. Manu. 62 (2012) 32-38.

[4] J.-H. Zhu, W.-H. Zhang, L. Xia, Topology Optimization in Aircraft Aerospace Structures and Design, Arch Computat. Methods Eng., (2015) $1-28$.

[5] R. Wauthle, J.P. Kruth, J. Schrooten, M. Mulier, Industrialization of Selective Laser Melting for the Production of Porous Titanium and Tantalum Implants, 120 pp. PhD, KU Leuven, 2014.

[6] B. Vandenbroucke, J.P. Kruth, Selective laser melting of biocompatible metals for rapid manufacturing of medical parts, Rapid Prototyp. J. 13 (2007) 196-203.

[7] N.W. Hrabe, P. Heinl, B. Flinn, C. Körner, R.J. Bordia, Compressioncompression fatigue of selective electron beam melted cellular titanium (Ti-6Al-4V). J. Biomed. Mater. Res. Part B 99 (2011) 313-320.

[8] S. Zhao, S.J. Li, W.T. Hou, Y.L. Hao, R. Yang, R.D.K. Misra, The influence of cell morphology on the compressive fatigue behavior of Ti-6Al-4V meshes fabricated by electron beam melting. J. Mech. Behav. Biomed. Mater. 59 (2016) 251-264.

[9] J.P. Kruth, G. Levy, F. Klöcke, T.H.C. Childs, Consolidation phenomena in laser and powder-bed based layered manufacturing, CIRP Ann.-Manuf. Techn. 56 (2007) 730-759.

[10] T. Niendorf, F. Brenne, M. Schaper, Lattice structures manufactured by SLM: on the effect of geometrical dimensions on microstructure evolution during processing, Metall. Mater. Trans. B 45 (2014) 11811185 .

[11] S.M. Ahmadi, S.A. Yavari, R. Wauthle, B. Pouran, J. Schrooten, H Weinans, A.A. Zadpoor, Additively Manufactured Open-Cell Porous Biomaterials Made from Six Different Space-Filling Unit Cells: The Mechanical and Morphological Properties, Materials 8 (2015) 1871-1896. 
[12] L.E. Murr, K.N. Amato, S.J. Li, Y.X. Tian, X.Y. Cheng, S.M. Gaytan, E. Martinez, P.W. Shindo, F. Medina, R.B. Wicker, Microstructure and mechanical properties of open-cellular biomaterials prototypes for total knee replacement implants fabricated by electron beam melting. J. Mech. Behav. Biomed. Mater. 4 (2011) 1396-1411.

[13] J. Kadkhodapour, H. Montazerian, A.C. Darabi, A.P. Anaraki, S.M. Ahmadi, A.A. Zadpoor, S. Schmauder, Failure mechanisms of additively manufactured porous biomaterials: Effects of porosity and type of unit cell, J. Mech. Behav. Biomed. Mater. 50 (2015) 180-191.

[14] S.M. Ahmadi, G. Campoli, S.A. Yavari, B. Sajadi, R. Wauthlé, J. Schrooten, H. Weinans, A.A. Zadpoor, Mechanical behavior of regular open-cell porous biomaterials made of diamond lattice unit cells, J. Mech. Behav. Biomed. Mater. 34 (2014) 106-115.

[15] S.A. Yavari, R. Wauthlé, J. van der Stok, A.C. Riemslag, M. Janssen, M. Mulier, J.P. Kruth, J. Schrooten, H. Weinans, A.A. Zadpoor, 2013. Fatigue behavior of porous biomaterials manufactured using selective laser melting, Mat. Sci. Eng. C 33 (2014) 4849-4858.

[16] S.A. Yavari, S.M. Ahmadi, R. Wauthle, B. Pouran, J. Schrooten, H. Weinans, A.A. Zadpoor, Relationship between unit cell type and porosity and the fatigue behavior of selective laser melted meta-biomaterials, J. Mech. Behav. Biomed. Mater. 43 (2015) 91-100.

[17] F. Li, J. Li, H. Kou, L. Zhou, Porous Ti6Al4V alloys with enhanced normalized fatigue strength for biomedical applications. Mater. Sci. Eng. C 60 (2016) 485-488

[18] S. Liu, H.Y. Du, Investigation on fatigue property of three dimensional reticulated porous metal foams, J. Mater. Sci. Technol. 28-5 (2012) 569-

575.

[19] M.F. Ashby, T. Evans, N.A. Fleck, J.W. Hutchinson, H.N.G. Wadley, L.J. Gibson, Metal foams: a design guide. Elsevier, 2000.

[20] A.M. Harte, N.A. Fleck, M.F. Ashby, Fatigue failure of an open cell and a closed cell aluminium alloy foam. Acta Mater. 47-8 (1999) 2511-2524.

[21] L.J. Gibson, M.F. Ashby, Cellular solids: structure and properties. Cambridge university press, Cambridge, 1999.

[22] A. Zargarian, M. Esfahanian, J. Kadkhodapour, S. Ziaei-Rad, Numerical simulation of the fatigue behavior of additive manufactured titanium porous lattice structures, Mat. Sci. Eng. C 60 (2016) 339-347.

[23] R. Hedayati, H. Hosseini-Toudeshky, M. Sadighi, M. MohammadiAghdam, A.A. Zadpoor, Computational prediction of the fatigue behavior of additively manufactured porous metallic biomaterials, Int. J. Fatigue 84 (2016) 67-79.

[24] B. Van Hooreweder, R. Boonen, D. Moens, J.P. Kruth, P. Sas, On the determination of fatigue properties of Ti6Al4V produced by selective laser melting. AIAA Structures, Structural Dynamics and Materials Conference. Honolulu, Hawaii, USA, 23-26 April 2012.

[25] B. Van Hooreweder, D. Moens, R. Boonen, J.P. Kruth, P. Sas, Analysis of fracture toughness and crack propagation of Ti6Al4V produced by selective laser melting, Adv. Eng. Mater. 14 (2012) 92-97.

[26] P.R. Onck, E.W. Andrews, L.J. Gibson, Size effects in ductile cellular solids. Part I: modeling, Int. J. Mech. Sci. 43 (2001) 681-699.

[27] E. W. Andrews, G. Gioux, P. Onck, L.J. Gibson, Size effects in ductile cellular solids. Part II: experimental results, Int. J. Mech. Sci. 43 (2001) 701-713.

[28] E. Yasa, J.P. Kruth, Application of laser re-melting on selective laser melting parts, Adv. Produc. Engineer. Manag. 6 (2011) 259-270.

[29] R. Wauthle, B. Vrancken, B. Beynaertts, K. Jorissen, J. Schrooten, J.P. Kruth, J. Van Humbeeck, Effects of build orientation and heat treatment on the microstructure and mechanical properties of selective laser melted Ti6Al4V lattice structures, Addit. Manuf. 5 (2015) 77-84.

[30] G. Pyka, A. Burakowski, G. Kerckhofs, M. Moesen, S. Van Bael, J. Schrooten, M. Wevers, Surface modification of Ti6Al4V open porous structures produced by additive manufacturing, Adv. Eng. Mate. 14 (2012) 363-370.

[31] A.B. Spierings, M. Schneider, R. Eggenberger, Comparison of density measurement techniques for additive manufactured metallic parts, Rapid Prototyping J. 17 (2011) 380-386.

[32] S.J. Li, L.E. Murr, X.Y. Cheng, Z.B. Zhang, Y.L. Hao, R. Yang, F. Medina, R.B. Wicker, Compression fatigue behavior of Ti6Al4V mesh arrays fabricated by electron beam melting, Acta Mater. 60 (2012) 793-802. [33] X.Y. Cheng, S.J. Li, L.E. Murr, Z.B. Zhang, Y.L. Hao, R. Yang, F. Medina, R.B. Wicker, Compression deformation behavior of Ti6Al4V alloy with cellular structures fabricated by electron beam melting, J. Mech. Behav. Biomed. Mater. 16 (2012) 153-162.

[34] B. Vrancken, L. Thijs, J.P. Kruth, J. Van Humbeeck, Heat treatment of Ti6Al4V produced by selective laser melting: microstructure and mechanical properties, J. Alloys Compd. 541 (2012) 177-185.
[35] C. Qiu, N.J.E. Adkins, M.M. Attallah, Microstructure and tensile properties of selectively laser-melted and of HIPed laser-melted Ti-6Al4V, Mater. Sci. Eng. A 578 (2013) 230-239.

[36] R. Wauthle, S.M. Ahmadi, S. Amin Yavari, M. Mulier, A.A. Zadpoor, H. Weinans, J. Van Humbeeck, J.-P. Kruth, J. Schrooten, Revival of pure titanium for dynamically loaded porous implants using additive manufacturing, Mater. Sci. Eng. C 54 (2015) 94-100.

[37] J. Schijve, Fatigue of structures and materials, Kluwer Academic, Dordrecht, 2001. 